\title{
Identification of monotonically differentially expressed genes for non-small cell lung cancer
}

Suyan Tian(D)

\begin{abstract}
Background: Monotonically expressed genes (MEGs) are genes whose expression values increase or decrease monotonically as a disease advances or time proceeds. Non-small cell lung cancer (NSCLC) is a multistage progression process resulting from genetic sequences mutations, the identification of MEGs for NSCLC is important.

Results: With the aid of a feature selection algorithm capable of identifying MEGs - the MFSelector method - two sets of potential MEGs were selected in this study: the MEGs across the different pathologic stages and the MEGs across the risk levels of death for the NSCLC patients at early stages. For the lung adenocarcinoma (AC) subtypes no statistically significant MEGs were identified across pathologic stages, however dozens of MEGs were identified across the risk levels of death. By contrast, for the squamous cell lung carcinoma (SCC) there were no statistically significant MEGs as either stage or risk level advanced.
\end{abstract}

Conclusions: The pathologic stage of non-small cell lung cancer patients at early stages has no prognostic value, making the identification of prognostic gene signatures for them more meaningful and highly desirable.

Keywords: Non-small cell lung cancer (NSCLC), Monotonically expressed genes (MEGs), Pathologic stages, Overall survival, Feature selection, Adenocarcinoma, Squamous cell carcinoma

\section{Background}

The 5-year survival rate of non-small cell lung cancer (NSCLC), which accounts for approximately $85 \%$ of lung cancer (LC) cases, remains very low [1]. So far, the most promising strategy of improving progression-free survival time and overall survival (OS) time of the NSCLC patients is early diagnosis followed by surgical resection, which is currently the standard of care. Unfortunately, at the time of diagnosis most NSCLC patients have already progressed to the advanced or metastatic stages, which are inoperable [2].

Since NSCLC is a multistage progression process resulting from genetic sequences mutations, researchers may be interested in knowing how the expression pattern of a gene varies as NSCLC progresses from early to late stages. This is especially the case with "monotonic" genes whose expression levels increase or decrease monotonically as

Correspondence: windytian@hotmail.com

Division of Clinical Research, The First Hospital of Jilin University, 71 Xinmin Street, Changchun 130021, Jilin, China the disease advances. Such an investigation may harvest no meaningful results given that no satisfactory segmentations among the early stages of NSCLC using gene expression profiles have yet been achieved [3-5]. Therefore, the likelihood of finding monotonically expressed genes (MEGs) across pathologic stages is extremely low.

Survival rates for stages I through IV of NSCLC decrease due to the progress of the disease such as the 5 -year survival rate for stage I is $47 \%$, stage II is $30 \%$, stage III is $10 \%$, and stage IV is $1 \%$ (http://www.cancer. org). These pathologic stages were determined according to the Cancer Staging System (http://cancerstaging.org) of the American Joint Committee on Cancer (AJCC). Nevertheless, for the NSCLC patients at the early stages of disease, the pathologic stage may not be a good index of how long a patient can expect to be progression-free and survive, given that a study by Der et al. [6] showed the hazard ratio for stage II versus stage I was 1.52 (95\% CIs: $0.9 \sim 2.55)$ and the corresponding $p$-value of the log-rank test which compared the survival curves of patients at stage I and stage II was 0.11. Meanwhile,

(c) The Author(s). 2019 Open Access This article is distributed under the terms of the Creative Commons Attribution 4.0 International License (http://creativecommons.org/licenses/by/4.0/), which permits unrestricted use, distribution, and 
continuous efforts [7-13] have been made to identify attributes predictive of progression-free survival or overall survival (mainly using gene expression profiles, thus an attribute corresponds to a gene), which may facilitate personalized medicine. Only with more "personalized" treatments that are tailored for a specific patient, could a stage I patient with poor prognosis live longer by receiving the adjuvant chemotherapy while a stage II patient with good prognosis could avoid suffering from adverse effects associated with the treatments and have a better quality of life.

Among NSCLC patients, adenocarcinoma (AC) and squamous cell carcinoma (SCC) are two major subtypes, accounting for roughly 40 and $35 \%$ of the lung cancer (LC) cases, respectively. Increasing evidence supports the fact that AC and SCC differ in many respects [14], therefore AC and SCC have been regarded as two distinct diseases. The DEGs for the two subtypes versus the normal controls naturally vary from each other. Hence, it is quite possible that MEGs across multiple pathologic stages for AC and SCC are distinct. Separate analyses for each subtype to identify their respective prognostic genes and respective MEGs are more appropriate than the analyses where these two subtypes are considered together as a whole.

In this study, a novel feature selection algorithm capable of identifying monotonically changed genes i.e., the monotonic feature selector (MFSelector) method [15] was used to test two research hypotheses. In [15], the authors demonstrated that the MFSelector method outperforms other competitive methods capable of identifying MEGs. The first hypothesis of the current study is to test if MEGs exist across different pathologic stages (i.e., stages IA, IB, IIA and IIB). The second hypothesis is to test the existence of MEGs over different risk profiles of a disease. Here, the risk levels of death were determined on the basis of the quartiles of overall survival time, namely, the extremely high-risk group whose survival time is less than $25 \%$ of survival time of all samples, the high-risk group is in between 25 and 50\%, the moderate-risk group is in between 50 and $75 \%$ and the low-risk group is above $75 \%$. My conjecture is that there are no MEGs across pathologic stages but some MEGs do exist over different risk levels. If this conjecture is true, it is more beneficial to predict a patient's risk of death using his/her gene expression profiles than using his/her pathologic stage alone, which provides more support for the necessity of finding genes with significant prognostic values for NSCLC patients at early stages.

\section{Methods}

\section{Experimental data}

The raw data of these two microarray experiments are publicly accessible on the GEO (https://www.ncbi.nlm. nih.gov/geo/) repository, under the accession numbers of GSE37745 [16] and GSE50081 [6]. All chips of these two experiments were profiled on the Affymetrix HGU133 Plus 2.0 platform. Only patients in these two cohorts who remained adjuvant treatment naïve with their clinical information such as survival time, age and smoking status available were included. Furthermore, in order to avoid ambiguous classification of a patient into a risk group (corresponding to the four quartiles of overall survival time), all censored patients were excluded from the downstream analyses, resulting in $73 \mathrm{AC} \mathrm{pa-}$ tients and 31 SCC patients included in this study. Among the $73 \mathrm{AC}$ patients, there were 11 stage IA, 39 stage IB, 5 stage IIA and 18 stage IIB patients. For SCC patients, there were 6 stage IA, 18 stage IB, 0 stage IIA and 7 stage IIB.

RNA-Seq data were downloaded from The Cancer Genome Atlas Data Portal (level 3) (https://tcga-data. nci.nih.gov/tcga/). Cohorts that were considered are: LUAD for the AC subtype and LUSC for the SCC subtype. By restricting selection to patients at early stages of disease and whose vital status was decreased, $73 \mathrm{AC}$ and 97 SCC patients were included in this analysis. Among the $73 \mathrm{AC}$ patients, there were 14 stage IA, 26 stage IB, 8 stage IIA and 23 stage IIB. For SCC patients, there were 17 stage IA, 46 stage IB, 12 stage IIA and 22 stage IIB. Notably, the adjuvant treatment restriction has been released for the RNA-Seq data since with it, there were only $17 \mathrm{AC}$ and 9 SCC patients left.

\section{Pre-processing procedures}

Raw data (CEL files) of these two microarray datasets were downloaded from the GEO repository. The expression values were obtained using the fRMA algorithm [17] and were normalized using quantile normalization. Then, the COMBAT algorithm [18] was used to eliminate or alleviate the batch effects existing between these two experiments. Counts-per-million (CPM) values for the RNA-seq data were calculated and $\log 2$ transformed by the Voom function in the R software.

\section{Monotonic feature selector}

The monotonic feature selector (MGSelector) method proposed by Wang et al. [15] introduces a new novel index, namely, the $\mathrm{DE}_{\text {total }}$ (total discriminating error) score for each gene, with the objective of selecting genes with strong monotonically changed patterns over stages or time. In addition, the corresponding $p$-value and $q$-value (which adjusts for the multiple comparisons) of this score was calculated using permutation tests to determine the significance level of a gene. There are two sets of monotonically expressed genes, namely a monotonically increasing/ascending set and the monotonically decreasing/descending set. The MGSelector method was 
A

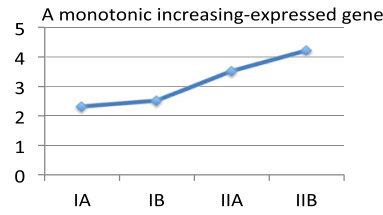

B

Property: $\quad \exp _{\mid \mathrm{A}}<\exp _{\mid \mathrm{B}}<\exp _{\| \mathrm{A}}<\exp _{\| \mathrm{BB}}$

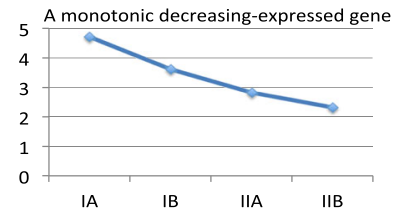

$\exp _{I A}>\exp _{I B}>\exp _{\| A}>\exp _{\| B}$
Step 1

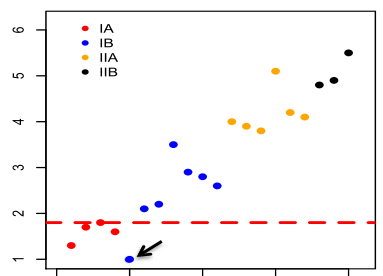

IA VS (IB, IIA, IIB)

error $=1$
Step 2

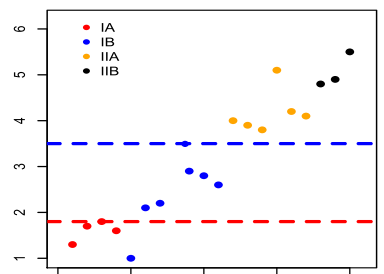

(IA, IB) VS (IIA, IIB)

error $=0$

$D E_{\text {total }}=2$
Step 3

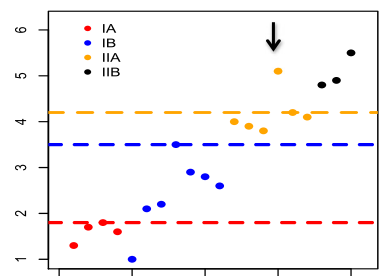

(IA, IB, IIA) VS IIB

error $=1$

Used permutation tests ( $N$ times permutations of labels) to obtain $p$-value/q-value for $D E_{\text {total }}$

Fig. 1 Graphical illustrations on what a MEG is and the MFSelector method. a The definition for a MEG. $\mathbf{b}$ The flowchart of the MFSelector method

described briefly as below using the ascending set as an example.

Suppose there are K levels/time points, it is natural to expect subjects in the lower levels have smaller expression values compared to the subjects in the remaining levels for a monotonically increasing expressed gene. First, $\mathrm{n}_{1}$ (here, $\mathrm{n}_{1}$ is the number of patients at the first level) discriminating lines may be drawn over the expression value of each patient at the first level. The samples at level I above this line and the patients at other levels below this line were misclassified into their opposite classes, and the number of misclassified samples (i.e., "discriminating errors") was counted. The final discriminating line to differentiate the level I from the other levels corresponded to the line with the least number of discriminating errors. This step was repeated for $\mathrm{K}-1$ times to discriminate the patients at the first $\mathrm{k}(\mathrm{k}=1,2, \ldots \mathrm{K}-1)$ levels apart from the remaining patients, resulting in $\mathrm{K}-1$ discriminating lines. If a gene has $\mathrm{K}-1$ distinct discriminating lines and the lines for a higher level are above the lines for a lower level, the expression change pattern of this specific gene presents a perfect monotonically increasing expression tendency. For the monotonically decreasing expression pattern, the discriminating lines taking the reversed orders with the line for a higher level below the line for a lower level were made.

The $\mathrm{DE}_{\text {total }}$ score is the sum of discriminating errors for all these $\mathrm{K}-1$ segmentations. Given the $\mathrm{DE}_{\text {total }}$ score does not make any distributional assumption on the data, it is less sensitive to the biases caused by outliers.
Table 1 The top-ranked genes across the pathologic stages by the MFSelector method

\begin{tabular}{|c|c|c|c|}
\hline \multicolumn{2}{|l|}{$A C$} & \multicolumn{2}{|l|}{ SCC } \\
\hline Ascending & Descending & Ascending & Descending \\
\hline DGCR14 * & AIMP1 & GRHL3 & CD97 \\
\hline SART1 * & CXADR & KIAA0907 & DUSP23 \\
\hline SLC34A1 * & socs6 & TM4SF1 & FBXW7 \\
\hline TYR * & TM2D1 & AKAP7 & GKN2 \\
\hline $\mathrm{CDH} 4{ }^{*}$ & ZBTB43 & CFL1 & PAG1 \\
\hline CNTROB ${ }^{*}$ & ALCAM & DBF4B & PAX5 \\
\hline CRYBA ${ }^{*}$ & ATP5L & F11R & RILPL2 \\
\hline GCK $^{*}$ & DYNC2H1 & PHF12 & SFTPB \\
\hline L1CAM * & PARD6B & PIP5K1A & TNNC1 \\
\hline LILRA3 * & POLI & RGS7 & TSPAN1 \\
\hline MATN4 * & RAB22A & RPL8 & \\
\hline NPAS4 * & RND3 & TFAP2A & \\
\hline NR4A3 ${ }^{*}$ & RPS21 & & \\
\hline SCNN1A * & SCP2 & & \\
\hline SLC39A2 ${ }^{*}$ & UBE2E1 & & \\
\hline \multicolumn{4}{|l|}{ SMARCD3 ${ }^{*}$} \\
\hline \multicolumn{4}{|l|}{ STMN4 * } \\
\hline TPSAB1 ${ }^{*}$ & & & \\
\hline
\end{tabular}

Note: * the corresponding q-value of $\mathrm{DE}_{\text {total }}<0.1 ;{ }^{* *} \mathrm{q}$-value $<0.05 ; \mathrm{AC}$ : lung adenocarcinoma; SCC: lung squamous cell carcinoma; Ascending: a monotonically expressed gene in the increasing order; descending: a monotonically expressed gene in the decreasing order 
Furthermore, a $p$-value and/or a $\mathrm{q}$-value of the $\mathrm{DE}_{\text {total }}$ score were calculated using permutation tests to determine if a gene was a statistically significant MEG or not. Figure 1 provides a graphical illustration for the definition of a MEG and the flowchart of the MFSelector method.

\section{Biological relevance and gene set analysis}

The biological relevance of the identified AC-specific MEGs and SCC-specific MEGs was investigated by searching the GeneCards database [19]. Then, the gene set enrichment analysis were carried out using the String software [20].

\section{Statistical language and packages}

All statistical analyses were carried out in the R language version 3.3.3 (www.r-project.org), and the R codes of the MFSelector algorithm were downloaded from the following webpage (http://microarray.ym.edu.tw/tools/MFSelector).
Additionally, the Venn-diagrams were made with the aid of an online bioinformatics tool (http://bioinformatics.psb. ugent.be/webtools/Venn/).

\section{Results}

Integrated microarray data

MEGs across pathologic stages

Using the integrated microarray data and the MFSelector method, the MEGs across pathologic stages for the AC and SCC subtypes were separately identified. First, the AC patients and SCC patients were rearranged in an ascending order according to their pathologic stages, respectively.

The top-ranked genes are listed separately for the AC and SCC subtypes in Table 1, and then within each subtype the genes are listed separately for the ascending expressed pattern and the descending expressed pattern. From this table, it was observed that if the cutoff of the corresponding $p$-value was set at 0.05 , there were no

Table 2 The top-ranked genes across the risk levels of death by the MFSelector method

\begin{tabular}{|c|c|c|c|c|c|}
\hline \multicolumn{4}{|l|}{$A C$} & \multicolumn{2}{|l|}{ SCC } \\
\hline Ascending & & Descending & & Ascending & Descending \\
\hline$\overline{G N L 3 L^{* *}}$ & FKBP3 ${ }^{* *}$ & $\mathrm{RGS2}^{* *}$ & ARF6 $^{a}$ & MMP1 & ATIC \\
\hline RALY* & FRAT1 ${ }^{* *}$ & POLK $^{* *}$ & C21orf91 ${ }^{a}$ & S100A2 & SART3 \\
\hline TBC1D10A & $\mathrm{GRK}^{* *}$ & $\mathrm{SHOC2}{ }^{* *}$ & ELK3 ${ }^{a}$ & TP53AIP1 & CRLS1 \\
\hline CDK19 ${ }^{* *}$ & HINFP ${ }^{* *}$ & ADD3 ${ }^{* *}$ & EPS15 $5^{a}$ & DEFB1 & DST \\
\hline CRY2 ${ }^{* *}$ & INPP5K ${ }^{* *}$ & EXOC1 ${ }^{* *}$ & IBTK ${ }^{\mathrm{a}}$ & EIF2S1 & EMG1 \\
\hline EPHB2 ${ }^{* *}$ & KLF13 ** & KRAS $^{* *}$ & $\mathrm{KPNA5}^{\mathrm{a}}$ & HSPG2 & FES \\
\hline MARK4 ${ }^{* *}$ & $M C 4 R^{* *}$ & SLC4A1AP** & MTF2 ${ }^{a}$ & IBTK & HSP90AB1 \\
\hline NAT6 ${ }^{* *}$ & $\mathrm{MDH} 2{ }^{* *}$ & DMTF1 $^{* *}$ & MYL12A & PAK2 & PEBP1 \\
\hline PLEKHJ1 ${ }^{* *}$ & MKNK2 ${ }^{* *}$ & $\mathrm{FRYL}^{* *}$ & PPP1CBa & $\mathrm{SH} 2 \mathrm{D} 3 \mathrm{C}$ & PMS1 \\
\hline RAB1B ${ }^{* *}$ & NARF ${ }^{* *}$ & ITGAV $^{* *}$ & RUFY $2^{\mathrm{a}}$ & SPHK1 & PPP6C \\
\hline RHOT2 ${ }^{* *}$ & PACSIN2 ${ }^{* *}$ & KIF5B ${ }^{* *}$ & $\mathrm{RWDD}^{\mathrm{a}}$ & UBR7 & \\
\hline TMEM222 ** & PRKACA ** & $\mathrm{SOD} 2^{* *}$ & STX7 $7^{a}$ & & \\
\hline ANP32A ** & RARA ** & $\mathrm{USO}^{* *}$ & $\mathrm{TBK} 1^{\mathrm{a}}$ & & \\
\hline $\mathrm{CORO} \mathrm{B}^{* *}$ & RFXANK ${ }^{* *}$ & & & & \\
\hline FNTB ${ }^{* *}$ & SIRT2 ${ }^{* *}$ & & & & \\
\hline MAP 7D1 ${ }^{* *}$ & STAT3 ** & & & & \\
\hline PI4KA ** & TACR2 ${ }^{* *}$ & & & & \\
\hline SHBG ${ }^{* *}$ & TMEM39B $^{* *}$ & & & & \\
\hline THRA ** & TPPP ** & & & & \\
\hline TINF2 ** & TSSC4 ${ }^{* *}$ & & & & \\
\hline UPF1 ${ }^{* *}$ & TUFM ${ }^{* *}$ & & & & \\
\hline ZNF142 ${ }^{* *}$ & URGCP ${ }^{* *}$ & & & & \\
\hline DLG5 ${ }^{* *}$ & WASF2 ${ }^{* *}$ & & & & \\
\hline EP400 ** & YIF1A ** & & & & \\
\hline
\end{tabular}

Note: ${ }^{*}$ the corresponding q-value of $\mathrm{DE}_{\text {total }}<0.1 ;{ }^{*} \mathrm{q}$-value $<0.05 ;{ }^{a} \mathrm{q}$-value $=0.05 ; \mathrm{AC}$ : lung adenocarcinoma; SCC: lung squamous cell carcinoma; Ascending: a monotonically expressed gene in the ascending order; descending: a monotonically expressed gene in the descending order. If the cutoff of q-value was set at 0.1 , there were 166 ascending MEGs and 172 descending MEGs for the AC subtype. However, no MEGs exist for the SCC subtype at the level of 0.1 
statistically significant MEGs for AC and SCC subtypes. If a less stringent threshold of 0.1 was chosen, only 18 genes in the AC ascending category were deemed to be statistically significant. As expected, no MEGs across pathologic stages can be identified if the cutoff for $p$-values/q-values is set at 0.05 , a predominant default value used in the field of statistics.

\section{MEGs across risk levels of death}

For this objective, the MFSelector method was used to identify MEGs across risk strata separately for the AC and SCC subtypes. Using the first quartile, the median and the third quartile of overall survival time for those $73 \mathrm{AC}$ patients as cutoffs, $\mathrm{AC}$ patients were divided into four groups with approximately 18 patients in each category. Likewise, SCC patients were also categorized into four groups with the number of patients in each group being approximately eight.

The most significant genes (with the least q-values) in this analysis are presented in Table 2. From this table, it was observed that for the AC subtype, 74 genes (48 were ascending expressed and 26 were descending expressed) were identified as the MEGs even with the more stringent cutoff (i.e., 0.05 for the q-value) being chosen. Nevertheless, for the SCC subtype, no genes were met with the less stringent cutoff of 0.1 , let alone 0.05 . The following two reasons may explain why no statistically significant MEGs were identified for this subtype. One is that the sample size of SCC patients in the experimental data was not big enough to have statistically significant results. The other possible explanation is the evolution of this disease is not a process of quantitative accumulation, implying the non-existence of MEGs indeed for the SCC subtype. Further investigation is warranted.

A Venn-diagram among these four categories is given in Fig. 2. There was only one overlapped gene, i.e., IBTK between the SCC \& risk and AC \& risk categories. The lack of overlapping between these two categories may imply two things. The first implication is that the identified MEGs are indeed subtype-specific. Therefore, either separate analyses for the AC and SCC subtypes or the utilization of statistical methods capable of identifying subtype-specific genes is highly recommended. The other is that the histological stage may not be a good representation of the risk status for overall mortality.

Besides the pathologic stage and the risk level of death, one clinical variable, i.e., a patient's age was considered. Similarly, using the first quartile, the median and the third quartile of age as cutoffs, both AC patients SCC patients were divided into four approximate equal-sized categories. For this index, no significant MEGs were identified for either AC subtype or SCC subtype, even at the less stringent cutoff for $p$-value of 0.1 .

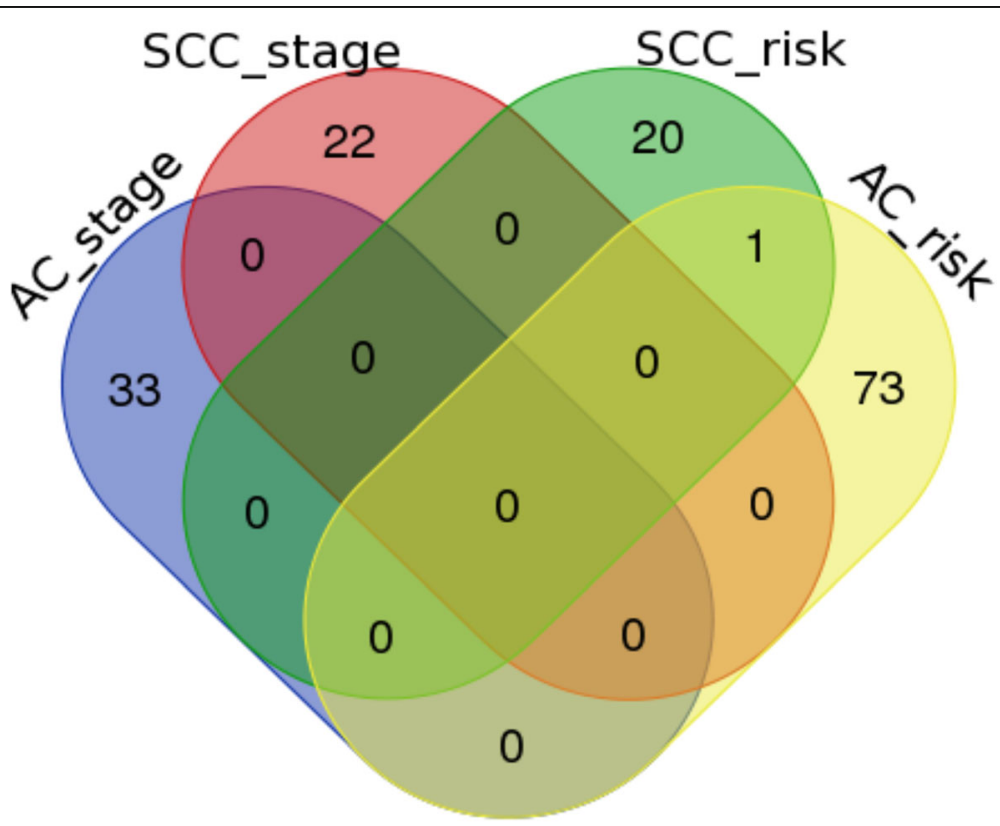

Fig. 2 Venn-diagram of the top-ranked genes among the four categories based on subtype and risk status/stage. AC_stage: across stages for lung adenocarcinoma subtype; SCC_stage: across stages for lung squamous cell carcinoma subtype; AC_risk: across risk levels of death for lung adenocarcinoma subtype; SCC_stage: across risk levels of death for lung squamous cell carcinoma subtype. There is only one overlapped gene between the SCC_risk and the AC_risk categories, i.e., IBTK that is indirectly related to lung cancer due to its association with KRAS, AKT1, BRAF and MAPK1 according to the GeneCards database 


\section{RNA-seq data}

Focusing on the pathologic stages and the risk levels of being death, the MFSelector method was applied to the RNA-seq data to find the MEGs. Then, a comparison between the MEGs identified using microarray data and the MEGs identified using RNA-seq data was made. Unfortunately, there were no statistically significant MEGs identified for all four categories - the AC \& stage category, the AC \& risk category, the SCC \& stage category and the SCC \& risk category at the level of 0.1 . Nevertheless, the top-ranked genes in the risk categories tended to have smaller $p$-values than those in the stage categories. For example, the corresponding $p$-value of the first-ranked descending gene for the SCC \& stage category was 0.149 whereas that of the descending gene on the top of the SCC \& risk category was only 0.116 .

Additionally, two other potential indices of the disease advancement were considered, namely, the number of packs of cigarettes smoked per year and the age at which the disease was diagnosed. Of note, such information is not available for the integrated microarray dataset. For both of these indices, no significant MEGs were identified for either AC or SCC at the level of 0.1.

Since the RNA-seq data included more hetero-generous patients in terms of adjuvant treatment option (including those that had adjuvant treatments and the status was unknown, in addition to those who remained adjuvant treatments naïve), it is unsurprising to observe the inconsistent results between the RNA-seq data and the microarray data.

\section{Discussion}

The biological relevance of those top-ranked genes listed on Tables 1 and 2 was searched in the GeneCards database. Focusing on the direct association supported by the literature, the searching results elucidated that for the AC \& stage category, only ALCAM, TYR and SART1 are related to lung adenocarcinoma but with very low confidence scores (1.60, 0.25 and 0.25 respectively). By contrast, for the AC \& risk category KRAS, STAT3, SOD2, KIF5B, ITGAV, EPHB2, RALY, ARF6, TBC1D10S, UPF1 and RARA are indicated to be directly related to lung adenocarcinoma. Among them, KRAS has the highest confidence score. It is well known that KRAS mutations are the most frequently oncogene aberrations in NSCLC patients [21, 22]. In this study, the identification of KRAS as a significant MEG points out a new direction to view the roles it may play during $\mathrm{AC}$ progression, from the perspective of expression values instead of somatic mutations. Likewise, STAT3 has a very high confidence score and many articles in the literature support its association with AC. For instance, a study by Qu et al. [23] stated that “... in both lung cancer and chronic obstructive pulmonary disease, the STAT3 gene was up-regulated” and “... STAT3 and its
Table 3 The overlapped GO terms

\begin{tabular}{|c|c|}
\hline Category & GO terms \\
\hline All & Cytosol \\
\hline \multirow[t]{13}{*}{ All but AC_risk } & translational termination \\
\hline & translational elongation \\
\hline & translational initiation \\
\hline & $\begin{array}{l}\text { nuclear-transcribed mRNA catabolic process, } \\
\text { nonsense-mediated decay }\end{array}$ \\
\hline & $\begin{array}{l}\text { SRP-dependent cotranslational protein targeting to } \\
\text { membrane }\end{array}$ \\
\hline & viral life cycle \\
\hline & viral transcription \\
\hline & cellular protein complex disassembly \\
\hline & viral gene expression \\
\hline & cytosolic ribosome \\
\hline & cellular macromolecule catabolic process \\
\hline & protein localization to membrane \\
\hline & focal adhesion \\
\hline \multirow[t]{4}{*}{ AC_stage \& SCC_risk } & structural constituent of ribosome \\
\hline & cellular component disassembly \\
\hline & mRNA metabolic process \\
\hline & Translation \\
\hline \multirow[t]{10}{*}{ SCC_risk \& SCC_stage } & establishment of protein localization to membrane \\
\hline & viral process \\
\hline & eyelid development in camera-type eye \\
\hline & large ribosomal subunit \\
\hline & cytosolic large ribosomal subunit \\
\hline & $\begin{array}{l}\text { establishment of protein localization to plasma } \\
\text { membrane }\end{array}$ \\
\hline & ribosome \\
\hline & intracellular protein transport \\
\hline & multi-organism process \\
\hline & protein targeting to membrane \\
\hline \multirow[t]{12}{*}{ AC_risk \& SCC_risk } & enzyme binding \\
\hline & purine ribonucleoside triphosphate binding \\
\hline & single-organism intracellular transport \\
\hline & purine ribonucleotide binding \\
\hline & single-organism transport \\
\hline & cytoplasmic transport \\
\hline & localization \\
\hline & kinase binding \\
\hline & purine ribonucleoside binding \\
\hline & nucleotide binding \\
\hline & cellular protein metabolic process \\
\hline & cellular protein localization \\
\hline
\end{tabular}

Note: AC stage represents across stages for lung adenocarcinoma subtype; SCC_stage represents across stages for lung squamous cell carcinoma subtype; AC_risk represents across risk levels of overall mortality for lung adenocarcinoma subtype; SCC_stage represents across risk levels of overall mortality for lung squamous cell carcinoma subtype 
A The KEGG pathways

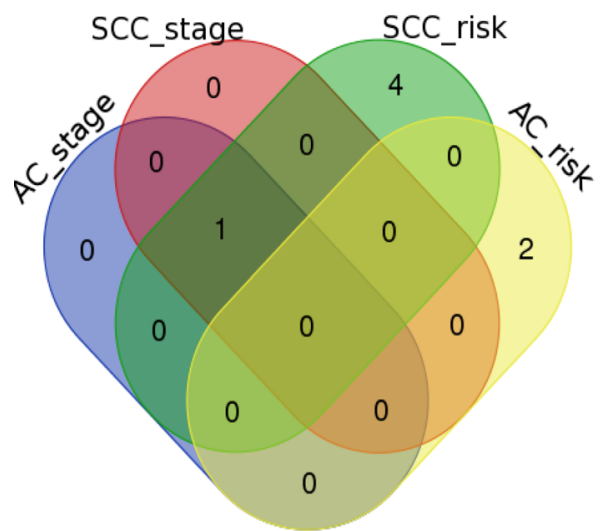

B The GO terms

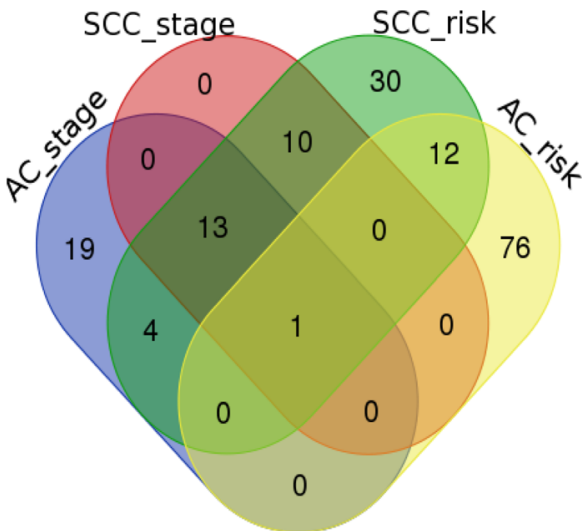

Fig. 3 Venn-diagram of the enriched KEGG pathways and GO terms between the four categories based on subtype and risk status/stage. a The enriched KEGG pathways; b The GO terms. Overall, the consistency level at the pathway level is higher. For the KEGG pathways, the ribosome pathway was commonly enriched in the AC_stage, SCC_stage and SCC_risk categories. For the GO terms, there is one term enriched by all four categories, 13 terms by all categories but the AC_risk category, 4 terms by the AC_stage and SCC_risk categories and 12 terms by the AC_risk and SCC_risk categories. AC_stage: across stages for lung adenocarcinoma subtype; SCC_stage: across stages for lung squamous cell carcinoma subtype; AC_risk: across risk levels of death for lung adenocarcinoma subtype; SCC_stage: across risk levels of death for lung squamous cell carcinoma subtype

downstream genes can serve as biomarkers for lung adenocarcinoma ..." . Therefore, many top-ranked genes in this category not only are statistically significant but also have meaningful biological interpretation.

For the SCC \& stage category, none of the top-ranked genes were found to correlate with SCC directly. On the other hand, three genes, i.e., PEBP1, S100A2 and HSP90AB1, are directly related to SCC for the SCC \& risk status category according to the GeneCards database. Even though the top-ranked genes identified in both categories are not statistically significant, the genes in the risk category have apparently better biological relevance. This is in consistent with the results for $\mathrm{AC}$, implying that histological stage may not be a good representative for the risk of death and thus may only have a limited prognostic value for the NSCLC patients at early stages.

As far as the enriched pathways were concerned, the degree of stability increased. The String software (www. string-db.org) indicated $89,37,81$ and 24 GO terms were enriched by the identified genes for the AC \& risk category, the AC \& stage category, the SCC \& risk category and SCC \& stage category, respectively. Among

\section{A}

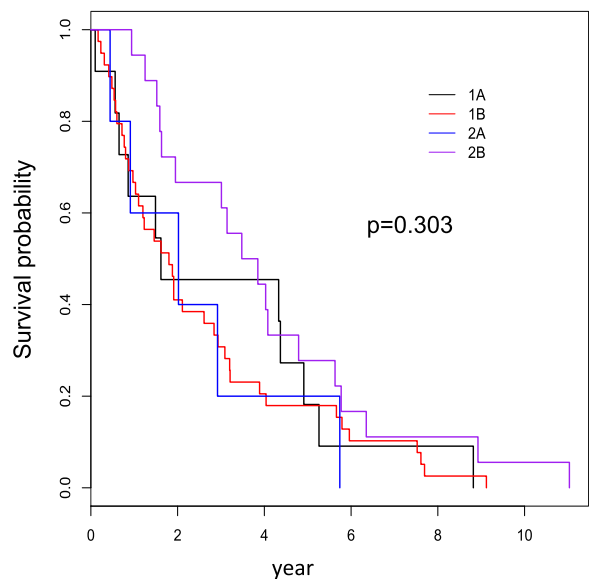

B The SCC subtype

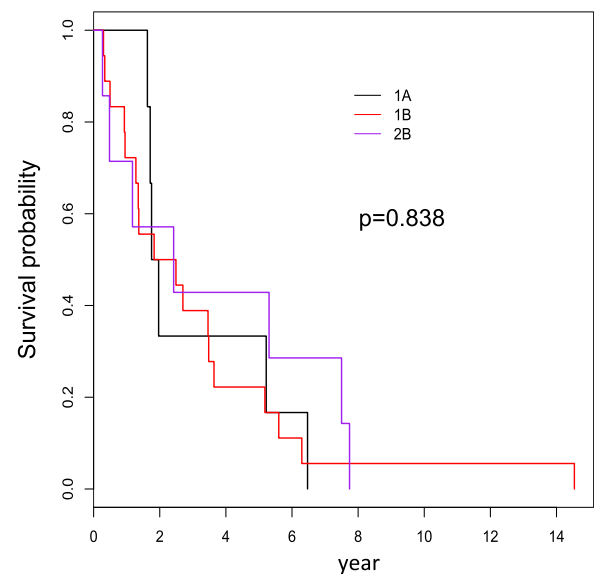

Fig. 4 Kaplan-Meier plots for the overall survival of non-small cell lung cancer stratified by the pathologic stages. a The AC subtype; b The SCC subtype. Here, $\mathrm{p}$ stands for the $p$-values of corresponding log-rank tests. AC: lung adenocarcinoma; SCC: lung squamous cell carcinoma 
165 unique enriched GO terms, only one term was commonly enriched by all four categories. Moreover, 13 terms were enriched by three categories and 14 terms were enriched by two categories, summing up to 40 overlapped terms. The $40(40 / 165 \cong 24.2 \%)$ overlapped GO terms are listed in Table 3. For the KEGG pathways, there were 2 enriched pathways for the AC \& risk category, 1 for AC \& stage, 5 for SCC \& risk and 1 for SCC \& stage. Only one overlapped pathway $(1 / 7 \cong 14.3 \%)$, the ribosome pathway, was commonly enriched in the AC \& stage, SCC \& stage and SCC \& risk categories. The Venn-diagrams of the KEGG pathways and the GO terms enriched in these four categories are given in Fig. 3. Although the overlapping proportion showed some increment compared to that at the gene level, it was still not substantially large. Therefore, the subtype-specific-gene conclusion remains true at the pathway level, namely the enriched pathways tend to be unique for $\mathrm{AC}$ and SCC subtypes.

To further explore whether the pathologic stage may serve as a surrogate for the risk of death, the Spearman's correlation coefficient between the risk level and the pathologic stage of patients was calculated, and Kaplan-Meier plots were made to compare the survival curves of different pathologic stages and log-rank tests were carried out to determine if the differences between those survival curves were statistically significant. The Spearman's correlation coefficient for the AC subtype was estimated as 0.19 with a $p$-value of 0.11 , and for the SCC subtype it was 0.02 with a p-value of 0.91 . Figure 4 presents the Kaplan-Meier plots, the p-value of the log-rank test for AC was 0.303, and for SCC it was 0.838 . Based on these tests, it was concluded that there is no significant association between pathologic stage and risk of death for the NSCLC patients at early stages.

\section{Conclusions}

With the aid of a feature selection algorithm capable of identifying MEGs, namely the MFSelector method [15], two research hypotheses were investigated. Using an integrated microarray dataset, the analyses showed that across pathologic stages there were no statistically significant MEGs whereas dozens of MEGs across risk levels of death were identified for the AC subtype. For the SCC subtype, however, there were no statistically significant MEGs in either stage or risk level categories. This may be explained by that either the sample size of the SCC subtype in this study is too small to guarantee an adequate statistical power or the disease progression of SCC differs.

The results of this study suggest that the pathologic stage is not well correlated with the risk of death and thus necessitate that the construction of prognostic gene signatures for NSCLC patients. More work is especially needed to develop more machine learning models capable of selecting subtype-specific prognostic genes readily and to boost the real-world applications of those models.

\section{Abbreviations}

AC: Lung adenocarcinoma; CPM: Counts-per-million; GEO: Gene Expression Omnibus; GO: Gene ontology; KEGG: Kyoto Encyclopedia of Genes and Genomes; MEG: Monotonically expressed gene; MFSelector: Monotonic feature selector; NSCLC: Non-small cell lung cancer; OS: Overall survival; SCC: Squamous cell lung carcinoma

\section{Acknowledgements}

I thank Ms. Donna Gilbreath for the English editing, and the academic editor and two referees for their time on handling and reviewing this manuscript.

\section{Funding}

This study was supported by a fund (No. 31401123) from the National Natural Science Foundation of China (NSFC). The NSFC plays no roles on the design of the study; collection, analysis, and interpretation of data; and on writing the manuscript.

\section{Availability of data and materials}

The raw data of two microarray experiments (their accession numbers are GSE37745 and GSE50081) were downloaded from the Gene Expression omnibus (GEO) repository (https://www.ncbi.nlm.nih.gov/geo/), and the RNASeq data of the LUAD (for AC) and LUSC (for SCC) cohorts were downloaded from The Cancer Genome Atlas (https://tcga-data.nci.nih.gov/tcga/). All data are publicly accessible.

\section{Author's contributions}

Conceived and designed the study: ST. Analyzed the data: ST. Interpreted data analysis and results: ST. Wrote the paper: ST. All authors reviewed and approved the final manuscript.

\section{Ethics approval and consent to participate}

Not applicable

Consent for publication

Not applicable

Competing interests

The author declares that he/she has no competing interests.

\section{Publisher's Note}

Springer Nature remains neutral with regard to jurisdictional claims in published maps and institutional affiliations.

Received: 5 March 2019 Accepted: 22 March 2019

Published online: 11 April 2019

\section{References}

1. Jemal A, Bray F, Center MM, Ferlay J, Ward E, Forman D. Global Cancer statistics: 2011. CA Cancer J Clin. 2011;61:69-90.

2. Sanchez-Palencia A, Gomez-Morales M, Gomez-Capilla JA, Pedraza V, Boyero $L$, Rosell R, Fárez-Vidal ME. Gene expression profiling reveals novel biomarkers in nonsmall cell lung cancer. Int J Cancer. 2011;129:355-64.

3. Tarca AL, Lauria M, Unger M, Bilal E, Boue S, Kumar Dey K, Hoeng J, Koeppl H, Martin F, Meyer P, Nandy P, Norel R, Peitsch M, Rice JJ, Romero R, Stolovitzky G, Talikka M, Xiang Y, Zechner C. Strengths and limitations of microarray-based phenotype prediction: lessons learned from the IMPROVER diagnostic signature challenge. Bioinformatics. 2013;29:2892-9.

4. Tian S, Chang HH, Wang C. Weighted-SAMGSR : combining significance analysis of microarray-gene set reduction algorithm with pathway topologybased weights to select relevant genes. Biol Direct. 2016;11:50.

5. Zhang L, Wang L, Du B, Wang T, Tian P, Tian S. Classification of non-small cell lung Cancer using significance analysis of microarray-gene set reduction algorithm. Biomed Res Int. 2016;2016:2491671. 
6. Der SD, Sykes J, Pintilie M, Zhu C, Strumpf D, Liu N, Jurisica I, Shepherd FA Tsao M-S. Validation of a histology-independent prognostic gene including stage IA patients. J Thorac Oncol. 2014;9:59-64.

7. Lu Y, Lemon W, Liu P-Y, Yi Y, Morrison C, Yang P, Sun Z, Szoke J, Gerald WL, Watson M, Govindan R, You M. A gene expression signature predicts survival of patients with stage I non-small cell lung cancer. PLoS Med. 2006;3:e467.

8. Tian S, Wang C, An M-W. Test on existence of histology subtype-specific prognostic signatures among early stage lung adenocarcinoma and squamous cell carcinoma patients using a cox-model based filter. Biol Direct. 2015;10:1-17.

9. Tian S, Wang $\mathrm{C}$, Chang HH, Sun J. Identification of prognostic genes and gene sets for early-stage non- small cell lung cancer using bi-level selection methods. Sci Rep. 2017:1-8.

10. Botling J, Edlund K, Lohr M. Biomarker discovery in non-small cell lung cancer : integrating gene expression profiling, meta-analysis and tissue microarray validation. Clin Cancer Res. 2013;19:194-204.

11. Tian S. Classification and survival prediction for early - stage lung adenocarcinoma and squamous cell carcinoma patients. Oncol Lett. 2017; 14:5464-70.

12. Krzystanek M, Moldvay J, Szüts D, Szallasi Z, Eklund AC. A robust prognostic gene expression signature for early stage lung adenocarcinoma. Biomarker Research. 2016:4:4

13. Liu W, Wu Y, Wang L, Gao L, Wang Y, Liu X, Zhang K, Song J, Wang H, Bayer TA, Glaser L, Sun Y, Zhang W, Cutaia M, Zhang DY, Ye F. Protein signature for non-small cell lung cancer prognosis. Am J Cancer Res. 2014;4(3):256-69.

14. Hou J, Aerts J, den Hamer B, van ljcken W, den Bakker M, Riegman P, van der Leest C, van der Spek P, Foekens JA, Hoogsteden HC, Grosveld F, Philipsen S. Gene expression-based classification of non-small cell lung carcinomas and survival prediction. PLoS One. 2010;5:e10312.

15. Wang H, Sun H, Chang T, Lo H, Cheng W, Tseng GC. Discovering monotonic stemness marker genes from time-series stem cell microarray data. BMC Genomics. 2015;16:S2.

16. Rousseaux S, Debernardi A, Jacquiau B, Vitte A, Vesin A, Nagy-mignotte $H_{\text {, }}$ Moro-sibilot D, Brichon P, Hainaut P, Laffaire J, De RA, Beer DG, Timsit F, Brambilla C, Brambilla E, Khochbin S. Ectopic activation of germline and placental genes identifies aggressive metastasis-prone lung cancers. Sci Transl Med. 2013:5:186ra66.

17. McCall MN, Bolstad BM, Irizarry RA. Frozen robust multiarray analysis (fRMA). Biostatistics Oxford England. 2010;11:242-53.

18. Johnson WE, Li C, Rabinovic A. Adjusting batch effects in microarray expression data using empirical Bayes methods. Biostatistics Oxford England. 2007;8:118-27.

19. Safran M, Dalah I, Alexander J, Rosen N, Stein TI, Shmoish M, Nativ N, Bahir I, Doniger T, Krug H, Sirota-madi A, Olender T, Golan Y, Stelzer G, Harel A, Lancet D. GeneCards Version 3 : the human gene integrator. Database. 2010:2010:baq020

20. Franceschini A, Szklarczyk D, Frankild S, Kuhn M, Simonovic M, Roth A, Lin J, Minguez P, Bork P, von Mering C, Jensen LJ. STRING v9.1: protein-protein interaction networks, with increased coverage and integration. Nucleic Acids Res. 2013:41:D808-15.

21. Román M, Baraibar I, López I, Nadal E, Rolfo C, Vicent S. KRAS oncogene in non-small cell lung cancer : clinical perspectives on the treatment of an old target. Mol Cancer. 2018;17:33.

22. Karachaliou N, Mayo C, Costa C, Magrí I, Gimenez-capitan A, Molina-vila MA, Rosell R. KRAS mutations in lung Cancer. Clinical Lung Cancer. 2013;14:205-14.

23. Qu P, Roberts J, Li Y, Albrecht M, Cummings OW, John N. Stat3 downstream genes serve as biomarkers in human lung carcinomas and chronic obstructive pulmonary disease. Lung Cancer. 2009;63:341-7.

\section{Ready to submit your research? Choose BMC and benefit from:}

- fast, convenient online submission

- thorough peer review by experienced researchers in your field

- rapid publication on acceptance

- support for research data, including large and complex data types

- gold Open Access which fosters wider collaboration and increased citations

- maximum visibility for your research: over $100 \mathrm{M}$ website views per year

At $\mathrm{BMC}$, research is always in progress.

Learn more biomedcentral.com/submissions 